Effect of the limiter position on the scrape-off layer width, radial electric field and intrinsic flows

This content has been downloaded from IOPscience. Please scroll down to see the full text.

View the table of contents for this issue, or go to the journal homepage for more

Download details:

This content was downloaded by: riccipaolo

IP Address: 128.178.125.98

This content was downloaded on 25/07/2014 at 06:08

Please note that terms and conditions apply. 


\title{
Effect of the limiter position on the scrape-off layer width, radial electric field and intrinsic flows
}

\author{
J. Loizu, P. Ricci, F.D. Halpern, S. Jolliet and A. Mosetto \\ École Polytechnique Fédérale de Lausanne (EPFL), Centre de Recherches en Physique des Plasmas (CRPP), \\ CH-1015 Lausanne, Switzerland \\ E-mail: joaquim.loizu@epfl.ch
}

Received 20 November 2013, revised 7 May 2014

Accepted for publication 23 June 2014

Published 24 July 2014

\begin{abstract}
The effect of the limiter position on the scrape-off layer (SOL) width, radial electric field and intrinsic flows is investigated via global, three-dimensional turbulence simulations in four different limiter configurations. The limiter position affects the SOL dynamics in a number of ways, for example by changing the effective connection length or by modifying the unstable modes present in the system. The simulations show that the SOL width is much smaller and less poloidally asymmetric when the plasma is limited on the low-field side than on the high-field side, which can be explained by a change in the turbulence regime between the two configurations. The radial electric field is determined by the combined effect of the sheath physics and the electron adiabaticity condition, and its poloidal structure depends on the limiter position, as it can be fairly well explained through an analytical model. Intrinsic parallel flows established in the SOL, typically leading to co-current toroidal rotation with a magnitude that strongly depends on the limiter position, can also be fairly well reproduced analytically for each limiter configuration.
\end{abstract}

Keywords: toroidal rotation, scrape-off layer, turbulence, flows, sheath, poloidal asymmetries

(Some figures may appear in colour only in the online journal)

\section{Introduction}

The plasma start-up in ITER will be in a limited configuration [1], using either the inner or outer vessel wall as the limiting surface. Since this part of the tokamak vessel is not designed to handle large particle and heat fluxes as are the divertor plates, the start-up scenario must be carefully tailored to minimize its power load. In the last few years, dedicated experiments have been conducted in a number of tokamaks in order to characterize the fluctuations and equilibrium profiles in the scrape-off layer (SOL) of limited plasmas [2-8]. Substantially large asymmetries have been observed between the different explored configurations, e.g. for inner wall limited (IWL) versus outer wall limited (OWL) [3, 4, 6]. A common conclusion is that poloidally asymmetric parallel flows are both a cause and a symptom of these differences [9]. Along with these experimental studies, numerical models have been used to get insights on the underlying physical mechanisms that lead to such asymmetries [10-13]. The common conclusion is that the inclusion of anomalous, ballooning-like cross-field transport is required in order to reproduce the general qualitative behaviour observed in the experiments. However, such models included the anomalous transport in an ad hoc manner, namely via transport coefficient asymmetries or arbitrary unphysical forces [3]. Only recently have first-principles fluid turbulence simulations been used to study poloidal asymmetries in the SOL [14], and in [15] the importance of the limiter position on SOL turbulence and flows was confirmed for the first time via first-principles gyro-fluid turbulence simulations.

In this paper, we present the results of global, threedimensional fluid simulations of SOL turbulence carried out with the GBS (Global Braginskii Solver) code [16], which self-consistently contains, in particular, the physics of ballooning modes, drift waves and losses at the sheaths. Four different limiter positions are considered: high-field side (HFS), low-field side (LFS), top and bottom. We focus on the effect of the limiter position on the SOL width, the electrostatic potential and the toroidal rotation. For each case we give a qualitative explanation for the differences observed in the simulation results, in terms of the character of turbulent transport and the effect that the limiter has on it.

We would like to notice that the SOL configuration considered herein is oversimplified with respect to the experiments (circular magnetic flux surfaces, no magnetic shear, cold ions, electrostatic, large aspect ratio, etc). As a matter of fact we do not target a quantitative comparison with experimental measurements, but rather an understanding 
of the fundamental mechanisms that explain the dependence of the SOL plasma dynamics on the limiter position. This is achieved thanks to global, flux-driven, full- $n$, threedimensional simulations of plasma turbulence in different limited SOL configurations, with first-principles turbulent transport and self-consistent sheath boundary conditions.

The paper is organized as follows. In section 2, we describe the GBS model equations and parameters used to simulate SOL turbulence in different limiter configurations. The effect of the limiter position on the SOL width, electrostatic potential and intrinsic toroidal rotation is analysed in sections 3, 4 and 5, respectively. The conclusions and the outlook are found in section 6 .

\section{The GBS model}

The GBS code has been developed in the last few years with the goal of simulating plasma turbulence in the tokamak SOL by evolving the full profiles of the various quantities with no separation between 'perturbations' and 'equilibrium' [16]. These simulations can explore the self-consistent evolution and structure of the plasma profiles in the presence of (i) plasma density and heat input from the core of the fusion machine, (ii) cross-field transport produced by plasma instabilities (interchange instability or drift waves, for example) and (iii) parallel losses at the sheaths where the magnetic field lines terminate on the walls.

An electrostatic drift-reduced fluid model is suitable to describe the SOL $[17,18]$ and it is used by GBS for the description of the plasma dynamics. In fact, the electron meanfree path is much smaller than the connection length, $\lambda_{e} \ll L_{\|}$, and trapped particles play a minor role since $v_{i, e}^{*} \gg 1$ [19]. Also, electrostatic low-frequency turbulence at a small wave number, $\omega \ll \omega_{c i}, k_{\perp} \rho_{s}<1$ and $k_{\|} L_{\|} \sim 1$, is believed to dominate the cross-field transport of particles and heat in the SOL, mainly driven by interchange-like modes such as resistive ballooning modes [20].

Based on the drift-reduced Braginskii equations [21], GBS describes the time evolution of the plasma density, $n$, the vorticity, $\omega$, the electrostatic potential, $\phi$, the ion and electron parallel velocities, $V_{\| i}$ and $V_{\| e}$, and the electron temperature $T_{e}$, according to the following equations, written here in the electrostatic, cold ion limit:

$$
\begin{aligned}
& \frac{\partial n}{\partial t}=-\frac{1}{B}\{\phi, n\}-\nabla_{\|}\left(n V_{\| e}\right) \\
&+\frac{2}{e B}\left[C\left(p_{e}\right)-e n C(\phi)\right]+S_{n}, \\
& \frac{e n}{B \omega_{c i}} \frac{\partial \omega}{\partial t}=-\frac{e n}{B \omega_{c i}}\{\phi, \omega\}-\frac{e n}{B \omega_{c i}} V_{\| i} \nabla_{\|} \omega+\nabla_{\|} j_{\|} \\
&+\frac{2 e}{B} C\left(p_{e}\right), \\
& m_{e} n \frac{\partial V_{\| e}}{\partial t}=-m_{e} n \frac{1}{B}\left\{\phi, V_{\| e}\right\}-m_{e} n V_{\| e} \nabla_{\|} V_{\| e}-\nabla_{\|} p_{e} \\
&+e n \nabla_{\|} \phi-0.71 n \nabla_{\|} T_{e}+e n v_{\|} j_{\|}-\frac{4}{3} \eta_{0 e} \nabla_{\|}^{2} V_{\| e} \\
&-\frac{2 \eta_{0 e}}{3 e B} \nabla_{\|}\left(5 C\left(T_{e}\right)+5 \frac{T_{e}}{n} C(n)+e C(\phi)\right), \\
& m_{i} n \frac{\partial V_{\| i}}{\partial t}=-m_{i} n \frac{1}{B}\left\{\phi, V_{\| i}\right\}-m_{i} n V_{\| i} \nabla_{\|} V_{\| i}-\nabla_{\|} p_{e},
\end{aligned}
$$

$$
\begin{aligned}
\frac{\partial T_{e}}{\partial t} & =-\frac{1}{B}\left\{\phi, T_{e}\right\}-V_{\| e} \nabla_{\|} T_{e}-\frac{2}{3} T_{e} \nabla_{\|} V_{\| e} \\
& +0.71 \frac{2}{3} \frac{T_{e}}{e n} \nabla_{\|} j_{\|}+\frac{2}{3 n} \chi_{\| e} \nabla_{\|}^{2} T_{e} \\
& +\frac{4}{3} \frac{1}{e B}\left[\frac{7}{2} T_{e} C\left(T_{e}\right)+\frac{T_{e}^{2}}{n} C(n)-e T_{e} C(\phi)\right]+S_{T}
\end{aligned}
$$

which are coupled to the Poisson equation $\nabla_{\perp}^{2} \phi=\omega$. Here $\omega_{c i}=e B / m_{i}$ is the ion gyrofrequency, $B$ is the magnetic field strength, $v_{\|}$is the parallel Spitzer resistivity, $\chi_{\| e}$ is the electron parallel heat diffusivity and $\eta_{0 e}$ is the electron viscosity [22]. Also, $S_{n}$ and $S_{T}$ are the density and temperature sources, and $j_{\|}=e n\left(V_{\| i}-V_{\| e}\right)$ is the parallel current. We notice that the Poisson brackets,

$$
\{\phi, f\} \equiv \boldsymbol{b} \cdot(\nabla \phi \times \nabla f),
$$

represent the convection of the quantity $f$ with the $\boldsymbol{E} \times \boldsymbol{B}$ drift. Here $\boldsymbol{b}=\boldsymbol{B} / \boldsymbol{B}$ is the unit vector in the direction of the magnetic field. Also, the terms containing the curvature operator,

$$
C(f) \equiv \frac{B}{2}\left(\nabla \times \frac{b}{B}\right) \cdot \nabla f,
$$

arise from the divergence of the $\boldsymbol{E} \times \boldsymbol{B}$ and diamagnetic drifts, which is nonvanishing for finite magnetic field curvature. We would like to remark that a fluid description of the SOL dynamics with a model based on the Braginskii equations may be limited to low-temperature regimes such as the L-mode. In fact, high-temperature events such as the edge localized modes (ELMs) observed in H-mode plasmas [23] may require more sophisticated fluid closures [24].

For the sake of simplicity, we consider a tokamak SOL with circular magnetic flux surfaces, no magnetic shear and a large aspect ratio. In order to describe such a configuration, we choose the coordinate system as follows. We define $z$ as the parallel coordinate and we denote the perpendicular coordinates with $x$ and $y, x$ being the radial coordinate, and $y$ the coordinate perpendicular to both $x$ and the magnetic field (figure 1). The coordinate system is such that $(y, x, z)$ is righthanded. In this coordinate system, we have

$$
\begin{gathered}
C=\frac{1}{R}\left(\sin \theta \frac{\partial}{\partial x}+\cos \theta \frac{\partial}{\partial y}\right), \\
\{f, g\}=\frac{\partial f}{\partial y} \frac{\partial g}{\partial x}-\frac{\partial g}{\partial y} \frac{\partial f}{\partial x}, \\
\nabla_{\perp}^{2}=\frac{\partial^{2}}{\partial x^{2}}+\frac{\partial^{2}}{\partial y^{2}}, \\
\nabla_{\|}=\frac{\partial}{\partial z},
\end{gathered}
$$

where $\theta$ is the poloidal angle defined so that $\theta=0$ at the LFS mid-plane. Since the pitch angle of the magnetic field is very small, $\alpha=\epsilon / q \ll 1(\epsilon$ is the inverse aspect ratio and $q$ is the safety factor), the $(x, y)$ plane almost coincides with the poloidal plane $(x, \theta)$.

The GBS model equations, equations $1-5$, are completed with a set of boundary conditions at the end of the field lines, 


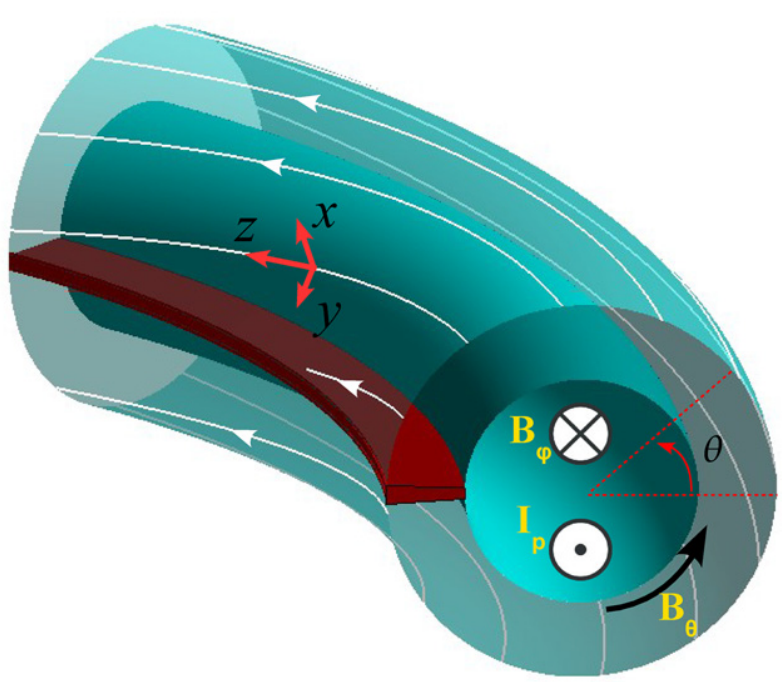

Figure 1. Sketch of the SOL geometry with its magnetic topology. The directions of the toroidal magnetic field $B_{\varphi}$, the poloidal magnetic field $B_{\theta}$, and that of the corresponding plasma current $I_{p}$ are indicated. Here the toroidal limiter is located on the HFS mid-plane $\left(\theta_{\text {limiter }}=\pi\right)$.

as given by the magnetic presheath entrance condition [25],

$$
\begin{aligned}
& V_{\| i}=c_{s}\left[ \pm 1+\theta_{n} \mp \frac{1}{2} \theta_{T_{e}}-2 \frac{e \phi}{T_{e}} \theta_{\phi}\right], \\
& V_{\| e}=c_{s}\left[ \pm \exp \left(\Lambda-e \phi / T_{e}\right)-2 \frac{e \phi}{T_{e}} \theta_{\phi}+2\left(\theta_{n}+\theta_{T_{e}}\right)\right],
\end{aligned}
$$

$\frac{\partial \phi}{\partial y}=-\frac{m_{i} c_{s}}{e}\left[ \pm 1+\theta_{n} \pm \frac{1}{2} \theta_{T_{e}}\right] \frac{\partial V_{\| i}}{\partial y}$,

$\frac{\partial n}{\partial y}=-\frac{n}{c_{s}}\left[ \pm 1+\theta_{n} \pm \frac{1}{2} \theta_{T_{e}}\right] \frac{\partial V_{\| i}}{\partial y}$,

$\frac{\partial T_{e}}{\partial y}=e \kappa_{T} \frac{\partial \phi}{\partial y}$

$$
\begin{aligned}
\nabla_{\perp}^{2} \phi & =-\frac{m_{i}}{e}\left[\left(1+\theta_{T_{e}}\right)\left(\frac{\partial V_{\| i}}{\partial y}\right)^{2}\right. \\
& \left.+c_{s}\left( \pm 1+\theta_{n} \pm \theta_{T_{e}} / 2\right) \frac{\partial^{2} V_{\| i}}{\partial y^{2}}\right],
\end{aligned}
$$

where the upper signs apply if the magnetic field is directed towards the wall, and the lower signs apply in the opposite case. Here $\Lambda=\log \left(\sqrt{m_{i} /\left(2 \pi m_{e}\right)}\right)$ and $\kappa_{T} \approx 0.1$ is a constant that comes from the analytical definition of the magnetic presheath entrance (see [25]). Also, in the considered geometry

$$
\theta_{f}=-\frac{\rho_{s}}{2 \tan \alpha} \frac{\partial_{x} f}{f}
$$

where $\rho_{s}=c_{s} / \omega_{c i}$ and $\alpha=\epsilon / q$ is the pitch angle of the magnetic field with respect to the wall surface. We remark that the electrostatic potential $\phi$ in equation (13) is measured with respect to the wall potential, which is assumed to be zero.

Finally, GBS works with normalized quantities defined through a reference temperature $\hat{T}_{e}$, a reference density $\hat{n}$ and a magnetic field $\hat{B}$. In particular, the electron temperature and the electrostatic potential are normalized such that $T_{e} \rightarrow T_{e} / \hat{T}_{e}$ and $\phi \rightarrow e \phi / \hat{T}_{e}$, and analogously for the density, $n \rightarrow n / \hat{n}$.

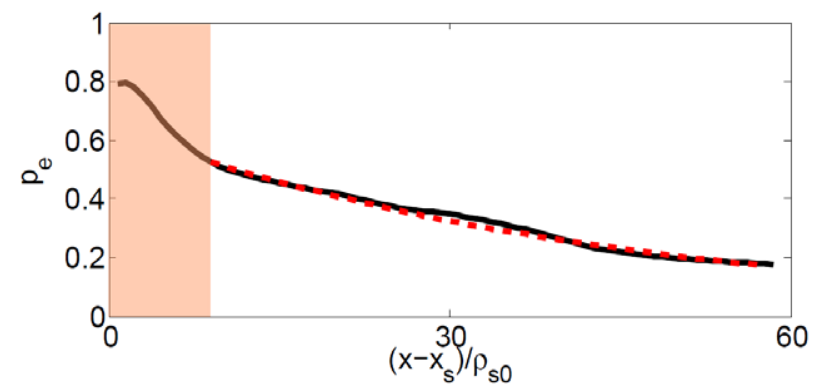

Figure 2. Example of equilibrium pressure profile on the LFS mid-plane $(\theta=0)$, for a simulation with the limiter on the HFS mid-plane $\left(\theta_{\text {limiter }}=\pi\right)$. The source radial extension is approximately $10 \rho_{s 0}$ (shaded area). In the source-free region, a reasonable fit of the radial profile can be obtained with an exponential function (dashed red line), leading to $L_{p} \simeq 45 \rho_{s 0}$.

The perpendicular coordinates are normalized with respect to $\hat{\rho}_{s}=\hat{c}_{s} / \hat{\omega}_{c i}$, where $\hat{c}_{s}=\sqrt{\hat{T}_{e} / m_{i}}$ and $\hat{\omega}_{c i}=e \hat{B} / m_{i}$. In the parallel direction, the macroscopic length $R$ is used. Finally, time is normalized such that $t \rightarrow t /\left(R / \hat{c}_{s}\right)$. The normalized system of equations evolved by GBS can be found in [16]. We remark that within this normalization, the parallel resistivity, heat diffusivity and viscosity are, respectively, normalized to $\hat{v}=m_{i} \hat{c}_{s} /\left(e^{2} \hat{n} R\right), \hat{\chi}=n \hat{T}_{e} R /\left(\hat{c}_{s} m_{i}\right)$ and $\hat{\eta}=n \hat{T}_{e}\left(R / \hat{c}_{s}\right)\left(m_{e} / m_{i}\right)$.

In this paper, we consider a fixed set of model parameters while changing the limiter position. These parameters are as follows: major radius $R=500 \hat{\rho}_{s}$, inverse aspect ratio $a / R \approx 0.25$, radial extension $L_{x}=x_{\max }-x_{\min }=100 \hat{\rho}_{s}$, safety factor $q=4$, mass ratio $m_{i} / m_{e}=200$, sheath coefficient $\Lambda=3$, parallel resistivity $v_{\|}=0.1 \hat{v}$, parallel heat diffusivity $\chi_{\| e}=\hat{\chi}$, and viscosity $\eta_{0 e}=\hat{\eta}$. We remark that the values of mass ratio and resistivity used in Ohm's law of these simulations are such that the resistive branch of ballooning modes and drift waves is expected to dominate over their inertial branch [26], thus they are consistent with the parameter regime usually explored experimentally. The angle between the magnetic field and the limiter is such that $\tan \alpha=a / q R \approx 0.0625$, corresponding to $\alpha \approx 3.6^{\circ}$. The particle and heat outflow from the core is modelled by density and temperature Gaussian sources that are radially localized at $x=x_{s}=x_{\min }+30 \hat{\rho}_{s}$ and have a width of $5 \hat{\rho}_{s}$. When analysing the simulation results only the region lying radially outwards from the source is considered. The peak of the source thus acts as the effective separatrix, defining the SOL region, and the region lying radially inwards is akin to a buffer region. Keeping fixed these model parameters, we consider four different limiter positions: LFS-limited $\left(\theta_{\text {limiter }}=0\right)$, HFS-limited $\left(\theta_{\text {limiter }}=\pi\right)$, top-limited $\left(\theta_{\text {limiter }}=\pi / 2\right)$ and bottom-limited $\left(\theta_{\text {limiter }}=-\pi / 2\right)$.

We now analyse the effect of the limiter position on the SOL width, electrostatic potential and intrinsic toroidal rotation.

\section{Effect of the limiter position on the SOL width}

The peak heat load onto the plasma-facing components of tokamak devices depends on the SOL width [7,27], which results from a balance between plasma injection from the core 


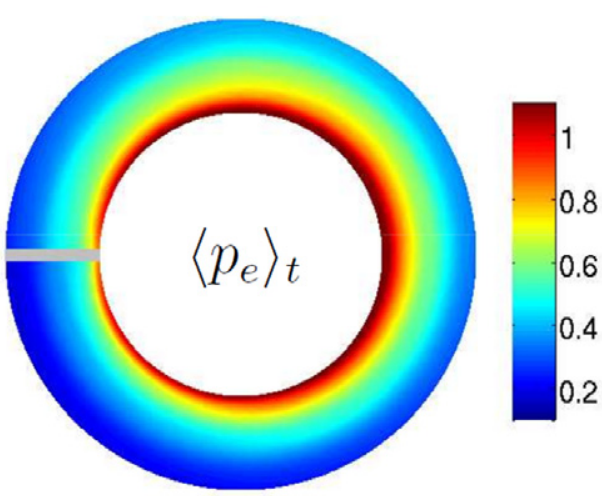

(a)

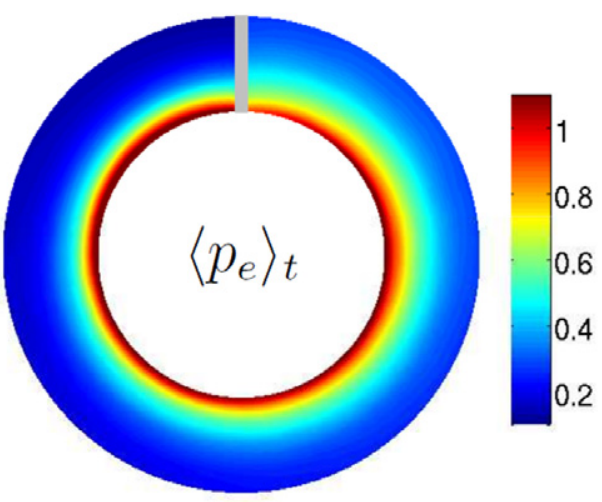

(c)

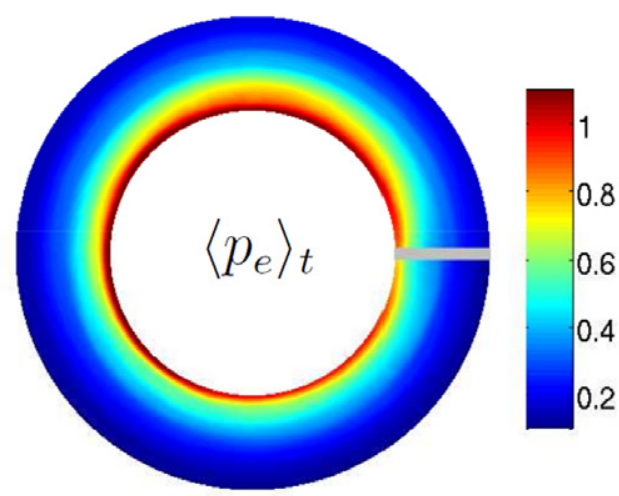

(b)

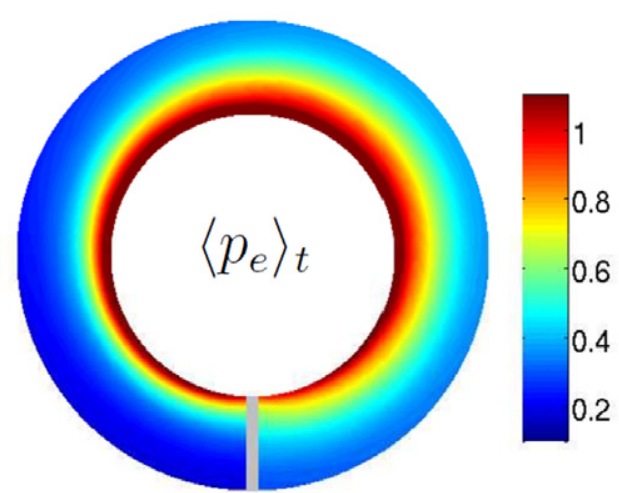

(d)

Figure 3. Equilibrium pressure profiles in a poloidal cross-section, with the limiter $(a)$ on the HFS equatorial mid-plane, $(b)$ on the LFS equatorial mid-plane, $(c)$ on the top of the vessel and $(d)$ on the bottom of the vessel. Results obtained from GBS simulations.

region, turbulent transport, and losses to the divertor or limiter plates [28]. Here we define the SOL width as the radial scale length of the plasma pressure,

$$
L_{p}=\left(\frac{1}{\bar{p}} \frac{\partial \bar{p}}{\partial x}\right)^{-1},
$$

which is computed from the simulation results by fitting, at each poloidal location, the equilibrium pressure profile with a radially decaying exponential function (overbar denotes timeaveraged quantities, while tilde denotes fluctuating quantities, e.g. $p=\bar{p}+\tilde{p}$ ), see figure 2 .

Figure 3 shows the equilibrium pressure profiles in a poloidal cross-section, for the four limiter configurations. The corresponding value of $L_{p}(\theta)$ is shown in figure 4. Clearly, the value of $L_{p}$ depends on the poloidal angle, $L_{p}=L_{p}(\theta)$, and it is affected by the limiter position. This is expected since the change in the limiter position modifies the effective connection length between the ballooning region and the limiter. We remark that the effect of flux expansion is not present in these simulations, thus the poloidal dependence of $L_{p}$ is only due to the poloidal asymmetries in the plasma turbulence and flows. First of all, we observe that the value of $L_{p}$ in the HFS-limited case (red curve in figure 4) is larger than that in the LFS-limited case (blue curve in figure 4), as observed experimentally [6]. The difference is a factor of 2 or less, while factors of 3 and higher have been obtained experimentally $[4,7]$. Second, in the four configurations considered, the values of $L_{p}$ tend to be

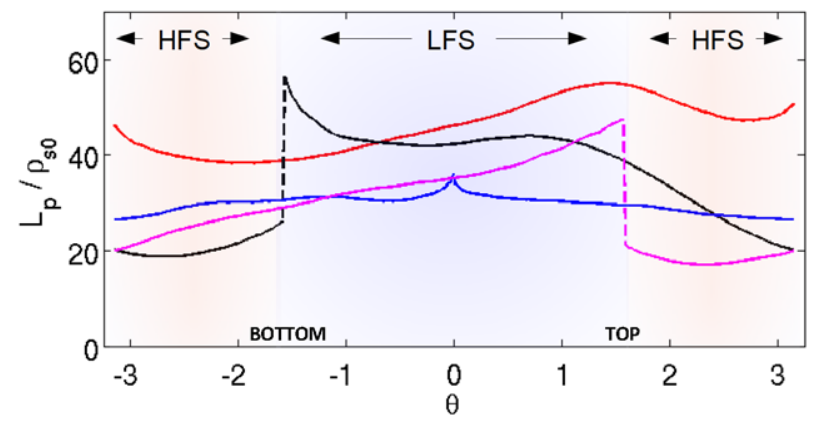

Figure 4. SOL width $L_{p}$ as a function of the poloidal angle, for a limiter on the HFS mid-plane (red), on the LFS mid-plane (blue), on the top of the vessel (magenta) and on the bottom of the vessel (black). Results obtained from GBS simulations.

larger on the LFS region than on the HFS region, consistent with the ballooning character of the turbulent transport. We notice, however, that this is marginally true for the HFS-limited configuration, where the peak of $L_{p}$ is shifted towards positive values of $\theta$. This shift is most likely due to the presence of an equilibrium $\boldsymbol{E} \times \boldsymbol{B}$ poloidal flow, which is positively directed along $\theta$. Also, in the LFS-limited configuration, the poloidal profile of $L_{p}$ is much flatter than in the other configurations, and this is due to the fact that turbulent transport is no longer dominated by ballooning modes. As a matter of fact, as we show now the poloidal dependence of $L_{p}$ is strongly affected 


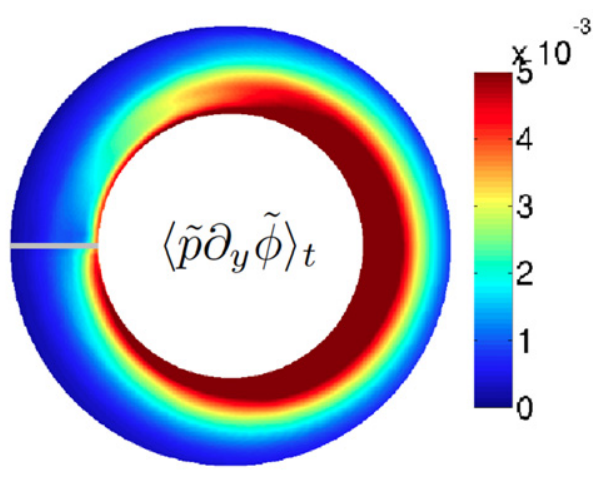

(a)

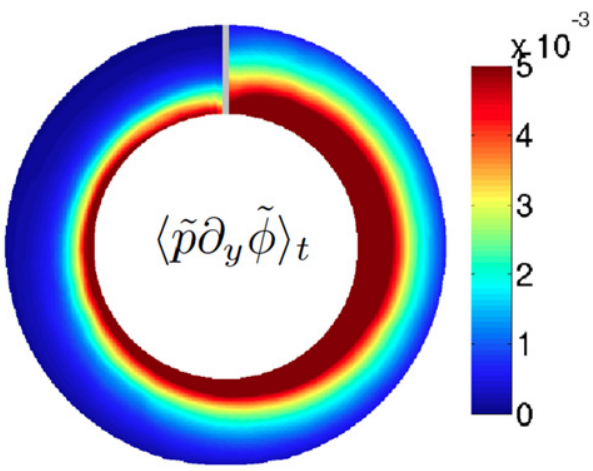

(c)

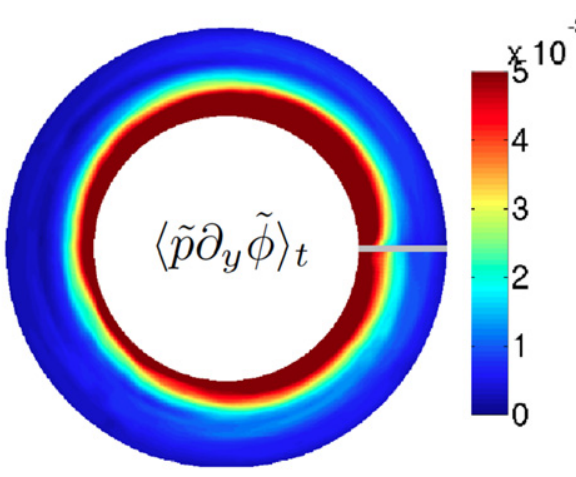

(b)

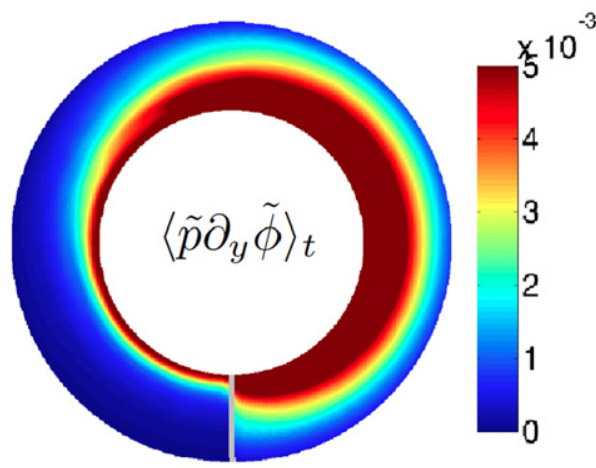

(d)

Figure 5. Time-averaged turbulent radial flux of plasma pressure in a poloidal cross-section and with the limiter $(a)$ on the HFS mid-plane, $(b)$ on the LFS mid-plane, $(c)$ on the top and $(d)$ on the bottom. Results obtained from GBS simulations.

by the turbulence properties, which in turn are affected by the limiter configuration. Figure 5 shows the time-averaged turbulent radial flux of plasma pressure, $\Gamma_{p}=\overline{\tilde{p} \partial_{y} \tilde{\phi}}$, in a poloidal cross-section and for the four limiter positions. In the HFS-limited, top-limited and bottom-limited configurations, the transport is clearly ballooned on the LFS. However, in the LFS-limited configuration, the transport is poloidally less asymmetrical; this is reflected in the weak dependence of $L_{p}$ on the poloidal angle, as shown in figure 4 .

These observations suggest that ballooning modes may become less efficient when the limiter is at the location of their maximum drive, thus steepening the pressure profiles. This suggests that transport may become drift-wave dominated, thus yielding more symmetric poloidal profiles. To assess this hypothesis, we compute the phase difference $\Delta \vartheta$ between density and potential fluctuations, which gives $\Delta \vartheta \simeq 0.4 \pi$ in the HFS-limited configuration and $\Delta \vartheta \simeq 0.1 \pi$ in the LFS-limited configuration. These are, respectively, consistent with the expected phase differences for ballooning modes $(\Delta \vartheta \simeq 0.5 \pi)$ and drift waves (small $\Delta \vartheta$ ). To further confirm this hypothesis, we proceed as follows. The value of $L_{p}$ can be predicted by using the gradient removal theory [18],

$$
L_{p} \sim \frac{q R}{c_{s}}\left(\frac{\gamma}{k_{y}}\right)_{\max },
$$

where $\gamma$ is the linear growth rate of the unstable mode present in the system that maximizes the ratio $\gamma / k_{y}$, with $k_{y}$ the poloidal wavenumber. Equation (20) results from a
Table 1. Values of the poloidally averaged pressure scale length $L_{p}$ predicted by the gradient removal theory, equation 20 (middle column), and obtained from nonlinear simulations (right column), for the HFS-limited and LFS-limited cases. The prediction of $L_{p}$ in the case of no interchange drive is also shown.

\begin{tabular}{lll}
\hline Limiter position & $\begin{array}{l}L_{p}\left[\rho_{s 0}\right] \\
\text { predicted }\end{array}$ & $\begin{array}{l}L_{p}\left[\rho_{s 0}\right] \\
\text { from GBS }\end{array}$ \\
\hline HFS & 40.2 & 44.2 \\
HFS (no interchange drive) & 27.5 & - \\
LFS (no interchange drive) & 29.9 & 29.4 \\
LFS (no in & - \\
\hline
\end{tabular}

pressure balance between parallel streaming and perpendicular turbulent transport. A detailed derivation of equation (20) is presented in the appendix.

The linear growth rate $\gamma$ that maximizes the ratio $\gamma / k_{y}$ is evaluated by using a linear code described in [26]. By finding numerically the value of $L_{p}$ that is consistent with equation (20), according to the procedure described in detail in [17], we obtain a predictive estimate of the value of the pressure scale length $L_{p}$ expected in nonlinear simulations. As a matter of fact, equation (20) has been used to estimate $L_{p}$ as a function of the SOL operational parameters, showing good agreement with experimental data from a number of tokamaks [28].

Table 1 shows the result of this procedure, which has been carried out for both HFS-limited and LFS-limited configurations. A reduction from $L_{p} \approx 40 \rho_{s 0}$ to $L_{p} \approx 30 \rho_{s 0}$ is expected when going from the HFS-limited configuration 


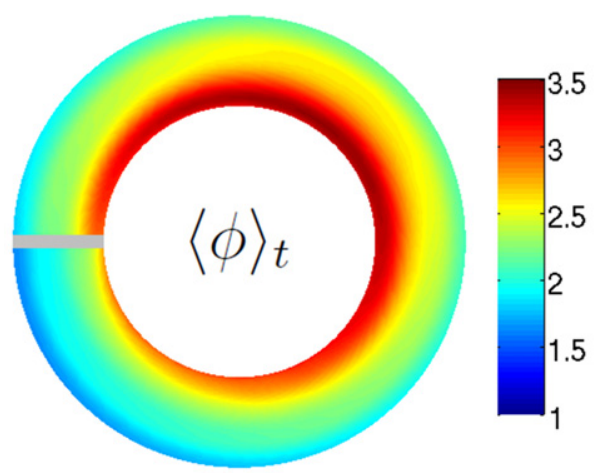

(a)

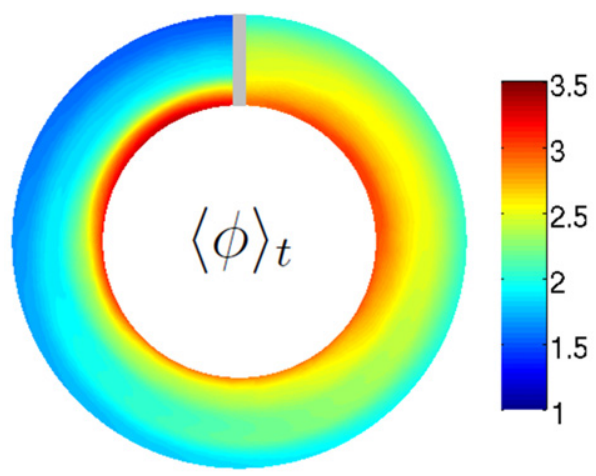

(c)

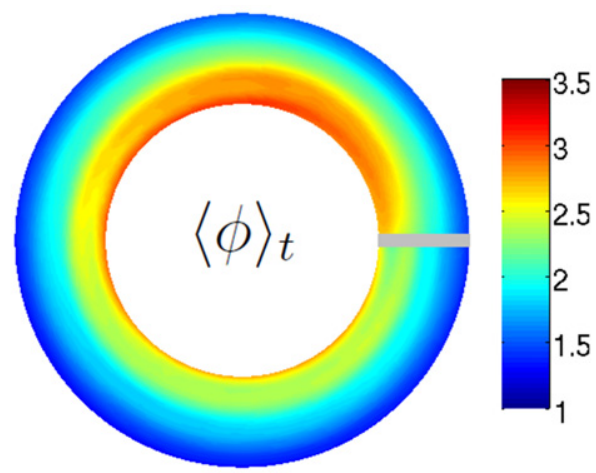

(b)

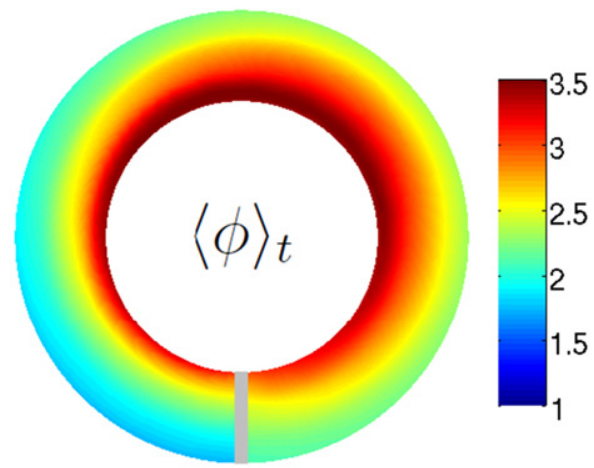

(d)

Figure 6. Equilibrium electrostatic potential profiles in a poloidal cross-section, with the limiter $(a)$ on the HFS mid-plane, $(b)$ on the LFS mid-plane, $(c)$ on the top and $(d)$ on the bottom. Results are obtained from GBS simulations.

to the LFS-limited configuration, which is in good agreement with the nonlinear simulation results shown in figure 4 and in table 1. Moreover, table 1 also shows the results obtained by following the same procedure but with the ballooning drive turned off (i.e. the curvature term in the vorticity equation, equation (2), is zeroed out). From the fact that turning off the ballooning drive only affects the value of $L_{p}$ in the HFS-limited case, we can deduce that the transport in the HFS-limited configuration is dominated by ballooning modes, while in the LFS-limited configuration, the transport is dominated by drift waves. This change in the turbulence regime is possibly due to the fact that for the LFS-limited case, the limiter is at the location of the maximum drive for the ballooning mode, and thus, by constraining its properties, the growth rate may be reduced.

\section{Effect of the limiter position on the electrostatic potential}

An essential quantity for the understanding of mean flows and pedestal formation during the $\mathrm{L}-\mathrm{H}$ transition in a magnetically confined plasma is the self-generated radial electric field. In addition, the radial electric field is important for the description of the heat losses and impurity dynamics. In this section, we examine the effect of the limiter position on the structure of the equilibrium electrostatic potential in the SOL poloidal plane.

In a recent paper [29], we show that the electrostatic potential in an open field line plasma configuration is set by the combined effect of two different mechanisms. On the one hand, the sheath physics regulates the value of $\phi$ at the end of the field lines to ensure quasi-neutrality in the main plasma. On the other hand, the electron adiabaticity sets the parallel electric field far from the walls. A general analytical relation between the equilibrium electrostatic potential and the equilibrium electron temperature and density, $\bar{\phi}=\bar{\phi}\left(\bar{T}_{e}, \bar{n}\right)$, was provided in [29] and is recalled here for convenience:

$$
\begin{aligned}
e \bar{\phi}= & \frac{1}{2} \Lambda\left(T_{e}^{+}+T_{e}^{-}\right)+1.71\left[\bar{T}_{e}-\frac{1}{2}\left(T_{e}^{+}+T_{e}^{-}\right)\right] \\
& +\sigma_{0}\left[\bar{n}-\frac{1}{2}\left(n^{+}+n^{-}\right)\right]
\end{aligned}
$$

where $T_{e}^{ \pm}$and $n^{ \pm}$represent, respectively, the temperature and density at the magnetic presheath entrance present at both ends of the magnetic field line, and $\sigma_{0} \simeq\left\langle\bar{T}_{e} / \bar{n}\right\rangle_{z}$, where $\langle\cdot\rangle_{z}$ denotes the average along the field line. The first term on the righthand side of equation (21) represents the effect of the sheath in determining the value of $\bar{\phi}$, while the second and third terms correspond to the effect of the plasma dynamics far from the walls.

The equilibrium profile of the electrostatic potential in a poloidal cross-section is shown in figure 6 for the four different limiter configurations. Strong poloidal asymmetries are clearly visible in each configuration, which means that the radial electric field varies significantly in the poloidal direction. As figure 7 shows, equation (21) can be used to describe the potential structure in the four limiter configurations. While the 


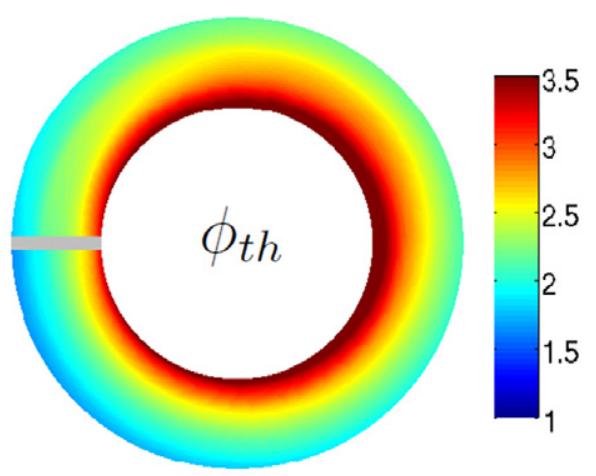

(a)

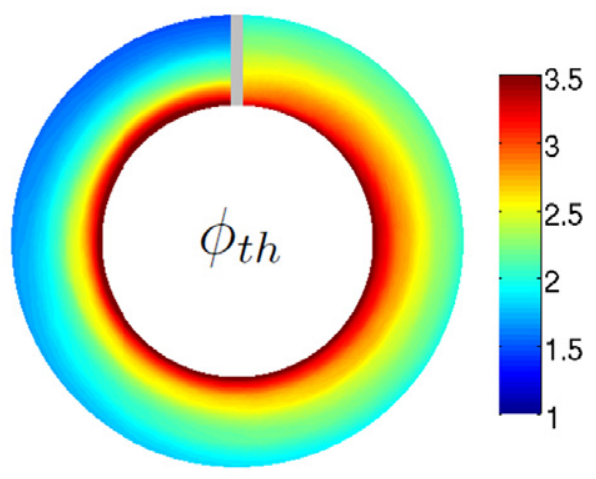

(c)

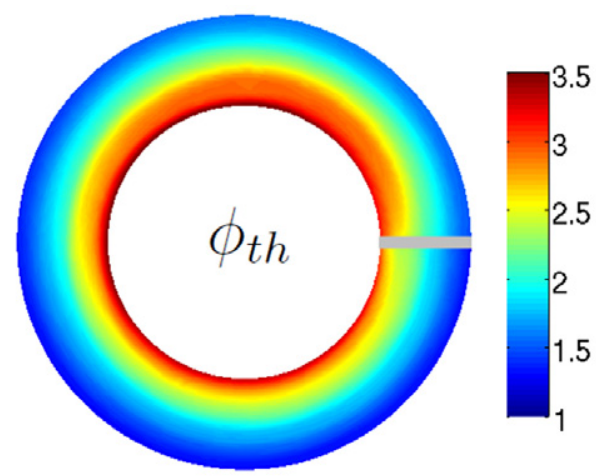

(b)

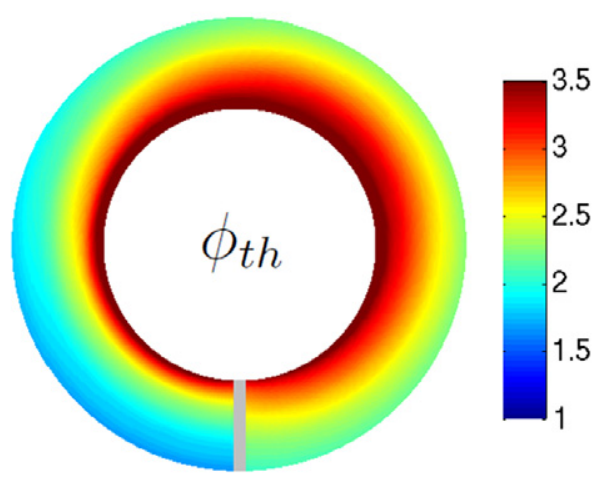

(d)

Figure 7. Equilibrium electrostatic potential profiles in a poloidal cross-section, with the limiter $(a)$ on the HFS mid-plane, $(b)$ on the LFS mid-plane, $(c)$ on the top and $(d)$ on the bottom. Results are obtained from equation (21).

model is able to reproduce both the magnitude and the radial and poloidal structure of the potential, we note that the poloidal asymmetries tend to be stronger in the simulations. This is due to the assumptions leading to equation (21), in particular the fact that the ratio of temperature to density is assumed to be about constant along the field line [29].

In general, the radial electric field is reduced in regions of enhanced transport, namely on the LFS, where the pressure profiles are also flatter. Moreover, since the equilibrium pressure profile depends on the position of the limiter, as shown in section 3, we expect from equation (21) that the equilibrium electrostatic potential is also affected by a change in the limiter position. The main effect is that the equilibrium scale length of the potential, $L_{\phi}$, is larger in the HFS-limited case $\left(L_{\phi} \approx 110 \rho_{s}\right)$ than in the LFS-limited case $\left(L_{\phi} \approx 80 \rho_{s}\right)$, as observed for $L_{p}$, and this is due to a change in the turbulence regime. Also, poloidal asymmetries are stronger in the HFSlimited, top-limited and bottom-limited configurations, where the transport is dominated by ballooning modes, than in the LFS-limited configuration, where it is dominated by drift waves.

\section{Effect of the limiter position on intrinsic toroidal rotation}

Recently, it has been found that strong flows in the SOL set the boundary conditions on the confined plasma and can even determine the low-to-high confinement mode power threshold [30]. The generation and transport of such flows has been investigated in [31] both analytically and through the use of numerical simulations. In particular, an analytical expression has been provided for the equilibrium profile of the toroidal Mach number $M(x, y)$ in the poloidal plane, where $x=0$ defines the separatrix and $y= \pm L_{y} / 2$ defines the two ends of the field line. This expression is recalled here:

$$
\begin{aligned}
& M(x, y)=M_{s} \mathrm{e}^{-x / l}+\left(M_{\mathrm{sh}}+M_{a}\right)\left(1-\mathrm{e}^{-x / l}\right)-2 \sigma_{\varphi} \frac{y}{L_{y}} \\
& +4\left[\left(M_{\mathrm{sh}}+M_{a}-M_{s}\right) \mathrm{e}^{-x / l}-M_{a}\right] \frac{y^{2}}{L_{y}^{2}},
\end{aligned}
$$

where $M=-\sigma_{\theta} \sigma_{\varphi} \bar{V}_{\| i} / c_{s 0}$ is the parallel Mach number projected in the toroidal direction along the plasma current $I_{p}$, such that $M>0$ always means co-current toroidal rotation. The subscript 0 denotes the value at $y=0$, and $\sigma_{\varphi}= \pm 1$ and $\sigma_{\theta}= \pm 1$ define the direction of the toroidal and poloidal components of the magnetic field, respectively, with the toroidal coordinate $\varphi$ defined in the counterclockwise direction when looking from the top of the machine. Here $M_{s}=M(0,0)$ is the separatrix condition half way between the two limiter sides or divertor legs, $M_{\mathrm{sh}}=\Lambda \rho_{s 0} /\left(\alpha L_{T}\right) \sim$ $\mathrm{e}^{-x / 2 L_{T}}$ represents the effect of the sheath on plasma rotation, and $M_{a}=\sigma_{\varphi}(\delta n+\delta T) / 2$ is due to the pressure poloidal asymmetry. $L_{T}$ is the radial scale length of the equilibrium temperature profile and $l=\lambda^{2} /\left(4 L_{T}\right)+\sqrt{\lambda^{2}+\left(\lambda^{2} /\left(4 L_{T}\right)\right)^{2}}$, 


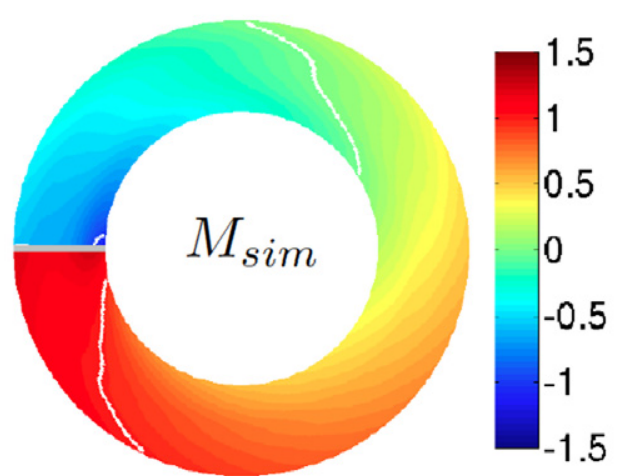

(a)

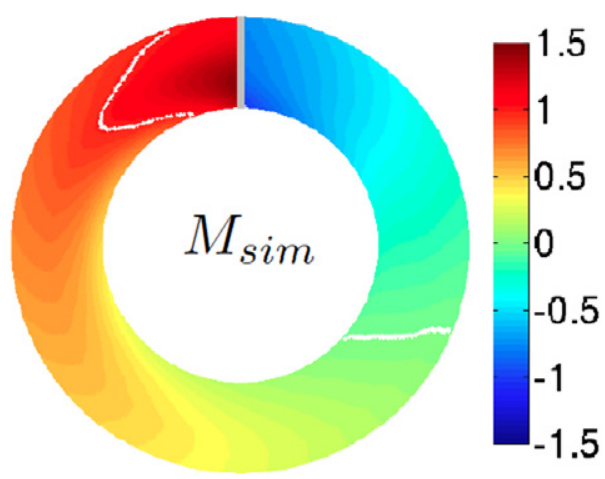

(c)

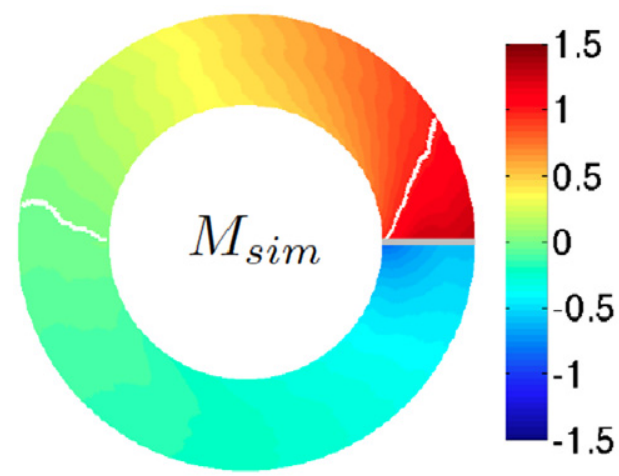

(b)

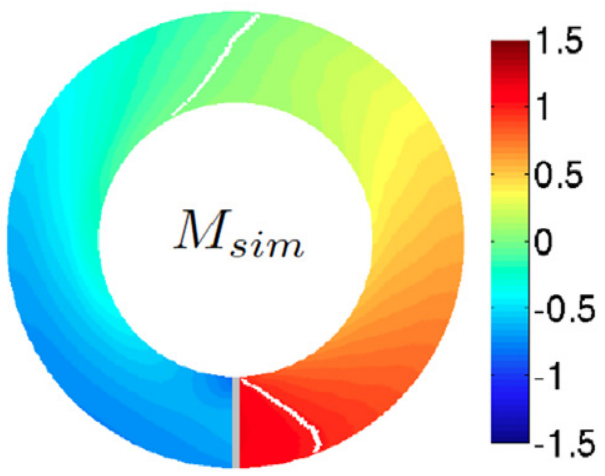

(d)

Figure 8. Equilibrium Mach number profiles $(M>0$ means co-current) in a poloidal cross-section, with the limiter $(a)$ on the HFS mid-plane, $(b)$ on the LFS mid-plane, $(c)$ on the top and $(d)$ on the bottom. The $M=0$ and $M= \pm 1$ iso-countours are shown in white (when present). Results obtained from GBS simulations.

where $\lambda^{2} \approx \pi L_{p}^{2}$. Also, the density asymmetry term, $\delta n$, can be estimated as $\delta n \sim\left(n^{+}-n^{-}\right) / n_{0}$, and similarly for $\delta T$.

Figure 8 summarizes the effect of the limiter position on the equilibrium profile of the Mach number in a poloidal cross-section. Clearly, both the radial and poloidal structure of the Mach number profile depends on the limiter position. In particular, the $M=0$ and $M= \pm 1$ iso-countours show that the plasma flow is poloidally asymmetric and that it can become subsonic/supersonic in the vicinity of the limiter. The latter is due to a recirculation of the $\boldsymbol{E} \times \boldsymbol{B}$ flow in the parallel direction at the magnetic presheath entrance [29]. These profiles can be compared with the analytical prediction of equation (22), as shown in figure 9. While the analytical model shows some discrepancies with respect to the simulations, the main features of the Mach number profile are reasonably captured: magnitude, volume average and general structure. In fact, as shown in table 2, a volume average of $M$ shows, both from the simulations and from the model, the presence of intrinsic toroidal flows, despite the fact that no momentum is injected into the system (see equation (4)). Moreover, the more favourable co-current configurations are those with a limiter on the HFS and on the top. This can be explained by the difference in the sign of $\delta n$, which gives a co-current contribution to the Mach number when $\delta n>0$, and a counter-current contribution when $\delta n<0$ (see table 2). The mechanism determining the sign of $\delta n$ is, as a matter of fact, the ballooning character of turbulent transport, which leads to an enhanced plasma pressure around the LFS and therefore to a poloidal pressure asymmetry between the two sides of the limiter [31]. Therefore, the location of the limiter can have a strong effect on the net toroidal rotation established in the SOL. We remark that, according to equation (22), this effect should reverse if the toroidal magnetic field is reversed $\left(\sigma_{\varphi}=-1\right)$. In general, favourable co-current configurations are expected to be given by the condition $M_{a}>0$, namely $\sigma_{\varphi}(\delta n+\delta T)>0$. This has been shown to agree with the measured experimental trends for the flow reversal in tokamaks, in particular those observed in Alcator C-Mod [31,32].

\section{Conclusions and outlook}

In this paper, the effect of the limiter position on the SOL equilibrium profiles has been investigated via GBS simulations considering four different limiter configurations: a limiter on the high-field side, low-field side, top and bottom parts of the vessel.

The width of the SOL has a clear poloidal dependence, which is qualitatively explained by the ballooning character of turbulent transport. Also, the SOL width varies significantly with the limiter position as the effective connection length between the ballooning region and the limiter is modified. Consistent with experimental measurements in a number of tokamaks, the SOL width is reduced when going from a LFSlimited configuration to a HFS-limited configuration, although 


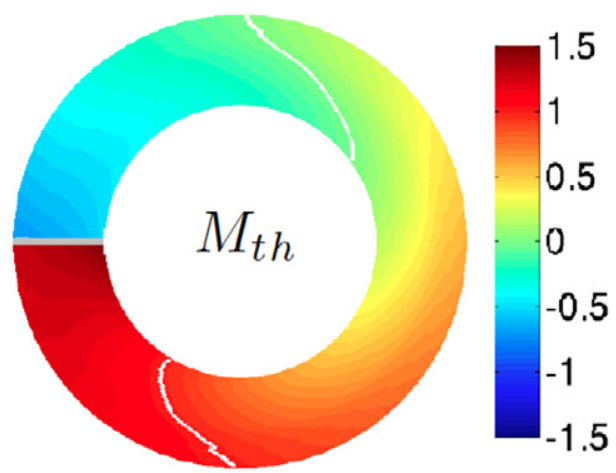

(a)

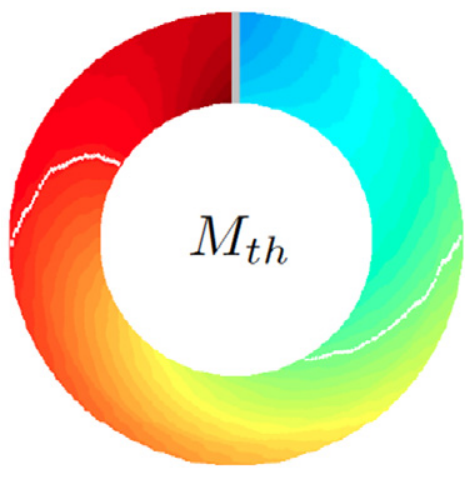

(c)

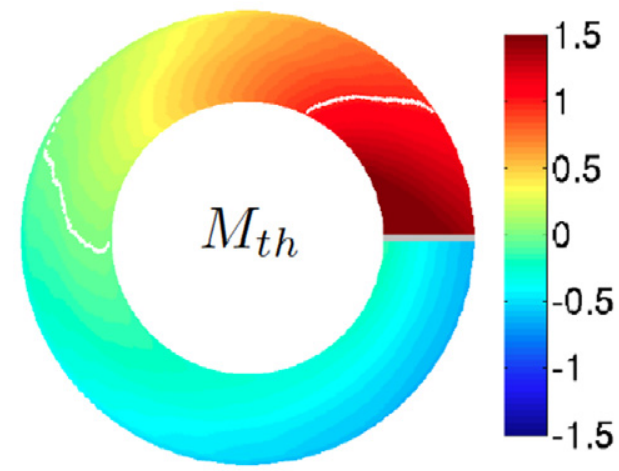

(b)

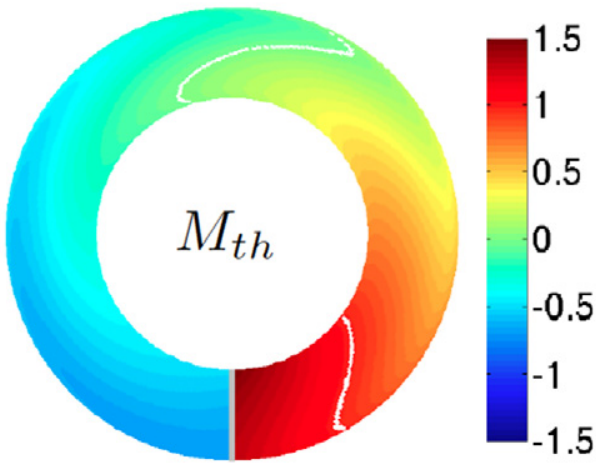

(d)

Figure 9. Equilibrium Mach number profiles ( $M>0$ means co-current) in a poloidal cross-section, with the limiter $(a)$ on the HFS mid-plane, $(b)$ on the LFS mid-plane, $(c)$ on the top and $(d)$ on the bottom. The $M=0$ and $M= \pm 1$ iso-countours are shown in white (when present). Results obtained from equation (22).

Table 2. Time and volume-averaged Mach number, $\langle M\rangle_{x, y}$, both from the simulations and from the theoretical model, and the corresponding sign of $\delta n$, for different limiter positions. For all cases $\sigma_{\varphi}=1$

\begin{tabular}{llll}
\hline Limiter position & $\left\langle M_{s i m}\right\rangle_{x, y}$ & $\left\langle M_{t h}\right\rangle_{x, y}$ & $\delta n$ \\
\hline LFS & 0.12 & 0.08 & $<0$ \\
HFS & 0.30 & 0.32 & $>0$ \\
TOP & 0.26 & 0.36 & $>0$ \\
BOT & 0.05 & 0.05 & $<0$ \\
\hline
\end{tabular}

the reduction is not as large as observed experimentally. We are able to show that this reduction is explained by a change in the turbulence regime. The transport in the HFS-limited configuration is dominated by ballooning modes, while in the LFS-limited configuration, the transport is dominated by drift waves. This is due to the location of the limiter, which has a stabilizing effect on the ballooning modes when its location coincides with the position of their maximum drive.

The limiter position also substantially modifies the equilibrium electrostatic potential and the intrinsic rotation profiles. The radial electric field is poloidally asymmetric, being reduced in the regions of enhanced transport, similarly to the pressure profile. Toroidal rotation profiles show co-current net toroidal flows, which can be strongly reduced depending on the limiter position. The analytical models developed in [29] and [31] are able to capture and explain the observed trends.

The results presented herein are obtained for a fixed set of SOL operational parameters. In particular, the tokamak major radius used here is much smaller than that of most tokamaks. Future work will focus on the dependences of these trends on the tokamak size $R$, the plasma resistivity $v_{\|}$and the magnetic shear $\hat{s}$, which can modify the relative importance of ballooning modes with respect to drift waves [17] and the magnitude of $L_{p}$ [28]. In particular, we anticipate that the difference in $L_{p}$ between HFS-limited and LFSlimited configurations may increase with the tokamak size. A detailed validation of our results with experimental data will be carried out.

Finally, we remark that the GBS simulations presented here are carried out in the so-called sheath-limited regime, where convection dominates over conduction. The future inclusion of neutral physics and diverted geometry may allow us to explore conduction-limited and detached regimes, where the pressure poloidal asymmetries are even stronger and therefore a larger effect on both the radial electric field and the toroidal Mach number is expected from equations (21) and (22).

\section{Acknowledgments}

We acknowledge useful discussions with A. Fasoli, I. Furno, C. Silva and G. Arnoux. A part of the simulations presented herein was carried out at the Swiss National Supercomputing Centre (CSCS) under project ID s346, and another part was carried out using the HELIOS supercomputer system at the 
Computational Simulation Centre of the International Fusion Energy Research Centre (IFERCCSC), Aomori, Japan, under the Broader Approach collaboration between Euratom and Japan, implemented by Fusion for Energy and JAEA. This work was supported by the Swiss National Science Foundation.

\section{Appendix}

Within the drift-reduced fluid model, a pressure continuity equation can be obtained by combining the density and temperature equations, equations (1) and (5). Assuming that the dominant terms are the parallel convection and the radial turbulent transport, we are left with an approximate pressure balance

$$
\frac{\partial p}{\partial t}+\frac{\partial \Gamma_{p}}{\partial x}+\nabla_{\|}\left(p V_{\| e}\right) \simeq 0
$$

where $\Gamma_{p}=\tilde{p} \partial_{y} \tilde{\phi} / B$ is the turbulent radial flux of plasma pressure. Writing

$$
{\overline{\partial_{x}}}_{p} \sim \bar{\Gamma}_{p} / L_{p}
$$

and

$$
\overline{\nabla_{\|}\left(p V_{\| e}\right)} \sim \bar{p} \bar{c}_{s} / q R,
$$

the time average of equation (A.1) leads to

$$
L_{p} \sim \frac{q R}{c_{s}} \frac{\bar{\Gamma}_{p}}{\bar{p}} .
$$

An estimate of $\bar{\Gamma}_{p}=\overline{\tilde{p} \partial_{y} \tilde{\phi}} / B$ can be obtained as follows. Linearizing equation (A.1) and keeping the dominant terms, one has

$$
\gamma \tilde{p} \sim \frac{1}{B} \frac{\partial \tilde{\phi}}{\partial y} \frac{\partial \bar{p}}{\partial x},
$$

thus relating the electric field fluctuations with the pressure fluctuations. Therefore, we have $\bar{\Gamma}_{p} \sim\left(\gamma L_{p} / \bar{p}\right) \overline{\tilde{p}^{2}}$. Finally, we can relate $\tilde{p}$ with $\bar{p}$ by using the gradient removal hypothesis, namely by assuming that the mode growth saturates when the fluctuations are able to remove the instability drive, which is provided by the pressure gradient, i.e. when

$$
\frac{\partial \tilde{p}}{\partial x} \sim \frac{\partial \bar{p}}{\partial x} .
$$

This condition can be written as $k_{x} \tilde{p} \sim \bar{p} / L_{p}$, where $k_{x}$ gives the radial extension of the saturated turbulent eddies. This can be estimated using nonlocal linear theory as $k_{x}=\sqrt{k_{y} / L_{p}}$ [33]. We can therefore write

$$
\overline{\tilde{p}^{2}} \sim \frac{\bar{p}^{2}}{k_{y} L_{p}} .
$$

Hence we are left with an expression for the radial pressure flux, $\bar{\Gamma}_{p}$, as a function of equilibrium quantities,

$$
\bar{\Gamma}_{p} \sim \bar{p}\left(\frac{\gamma}{k_{y}}\right)_{\text {max }},
$$

where the linear growth rate $\gamma$ and the wavenumber $k_{y}$ must be chosen in order to maximize the ratio of $\gamma / k_{y}$, thus maximizing the transport. Finally, we can replace this expression into equation (A.4), leading to

$$
L_{p} \sim \frac{q R}{c_{s}}\left(\frac{\gamma}{k_{y}}\right)_{\text {max }} .
$$

\section{References}

[1] Gribov Y., Humphreys D., Kajiwara K., Lazarus E.A., Lister J.B., Ozeki T., Portone A., Shimada M., Sips A.C.C. and Wesley J.C. 2007 Progress in the ITER Physics Basis: chapter 8. Plasma operation and control Nucl. Fusion 47 S385-403

[2] Hugill J. 1992 Interpretation of asymmetries in tokamak scrape-off layers in terms of poloidal variation of anomalous transport and diamagnetic effects $J$. Nucl. Mater. 196-198 918-22

[3] Gunn J.P. et al 2007 Evidence for a poloidally localized enhancement of radial transport in the scrape-off layer of the Tore Supra tokamak J. Nucl. Mater. 363-365 484-90

[4] Kocan M. and Gunn J.P. 2010 Comparison of scrape-off layer profiles in outboard-versus inboard-limited plasmas in Tore Supra Plasma Phys. Control. Fusion 52045010

[5] Rudakov D.L., Boedo J.A., Pitts R.A., Jackson G.L., Lasnier C.J., Leonard A.W., Moyer R.A., Stangeby P.C., Tynan G.R. and Watkins J.G. 2011 SOL width in limited versus diverted discharges in DIII-D J. Nucl. Mater. 415 S387-90

[6] Silva C., Arnoux G., Devaux S., Frigione D., Groth M., Horacek J., Lomas P.J., Marsen S., Matthews G. and Pitts R.A. 2013 Comparison of scrape-off layer transport in inner, outer wall limited JET plasmas J. Nucl. Mater. 438 S189-93

[7] Arnoux G. et al 2013 Scrape-off layer properties of ITER-like limiter start-up plasmas in JET Nucl. Fusion 53073016

[8] Silva C., Figuereido H., Duarte P. and Fernandes H. 2011 Characterization of the poloidal asymmentries in the ISTTOK edge plasma Plasma Phys. Control. Fusion 53085021

[9] Asakura N. 2007 Understanding the SOL flow in L-mode plasma on divertor tokamaks and its influence on the plasma transport J. Nucl. Mater. 363-365 41-51

[10] Pigarov A.Yu., Krasheninnikov S.I., Rognlien T.D., Schaffer M.J. and West W.P. 2002 Tokamak edge plasma simulation including anomalous cross-field convective transport Phys. Plasmas 91287

[11] Kirnev G.S., Corrigan G., Coster D., Erents S.K., Fundamenski W., Matthews G.F. and Pitts R.A. 2005 EDGE2D code simulations of SOL flows, in-out divertor asymmetries in JET $\mathrm{J}$. Nucl. Mater. 337-339 271-5

[12] Zagórski R., Gunn J.P. and Nanobashvili I. 2007 Numerical investigations of edge plasma flows in the Tore Supra tokamak Plasma Phys. Control. Fusion 49 S97-108

[13] Federici G. et al 2007 Simulations of ITER start-up, assessment of limiter power loads $J$. Nucl. Mater. 363-365 346-52

[14] Tamain P., Ghendrih Ph., Tsitrone E., Sarazin Y., Garbet X., Grandgirard V., Gunn J., Serre E., Ciraolo G. and Chiavassa G. 2009 3D modelling of edge parallel flow asymmetries J. Nucl. Mater. 390-391 347-50

[15] Ribeiro T.T. and Scott B. 2008 Gyrofluid turbulence studies of the effect of the poloidal position of an axisymmetric Debye sheath Plasma Phys. Control. Fusion 50055007

[16] Ricci P., Halpern F.D., Jolliet S., Loizu J., Mosetto A., Fasoli A., Furno I. and Theiler C. 2012 Simulation of plasma turbulence in scrape-off layer conditions: the GBS code, simulation results and code validation Plasma Phys. Control. Fusion 54124047

[17] Mosetto A., Halpern F.D., Jolliet S., Loizu J. and Ricci P. 2013 Turbulent regimes in the tokamak scrape-off layer Phys. Plasmas 20092308

[18] Ricci P. and Rogers B.N. 2013 Plasma turbulence in the scrape-off layer of tokamak devices Phys. Plasmas 20010702

[19] Rogers B., Drake J. and Zeiler A. 1998 Phase space of tokamak edge turbulence, the $\mathrm{L}-\mathrm{H}$ transition, and the 
formation of the edge pedestal Phys. Rev. Lett. 81 4396-9

[20] LaBombard B., Hughes J.W., Mossessian D., Greenwald M., Lipschultz B., Terry J.L. and the Alcator C-Mod Team 2005 Evidence for electromagnetic fluid drift turbulence controlling the edge plasma state in the Alcator C-Mod tokamak Nucl. Fusion 45 1658-75

[21] Zeiler A., Drake J.F. and Rogers B. 1997 Nonlinear reduced Braginskii equations with ion thermal dynamics in toroidal plasma Phys. Plasmas 42134

[22] Braginskii S. 1965 Transport processes in a plasma Rev. Plasma Phys. 1 205-311

[23] Wilson H.R., Cowley S.C., Kirk A. and Snyder P.B. 2006 Magneto-hydrodynamic stability of the H-mode transport barrier as a model for edge localized modes: an overview Plasma Phys. Control. Fusion 48 A71-84

[24] Omotani J.T. and Dudson B.D. 2013 Non-local approach to kinetic effects on parallel transport in fluid models of the scrape-off layer Plasma Phys. Control. Fusion 55055009

[25] Loizu J., Ricci P., Halpern F.D. and Jolliet S. 2012 Boundary conditions for plasma fluid models at the magnetic presheath entrance Phys. Plasmas 19122307
[26] Mosetto A., Halpern F.D., Jolliet S. and Ricci P. 2012 Low-frequency linear-mode regimes in the tokamak scrape-off layer Phys. Plasmas 19112103

[27] Makowski M.A. et al 2012 Analysis of a multi-machine database on divertor heat fluxes Phys. Plasmas 19056122

[28] Halpern F.D. et al 2013 Theory-based scaling of the SOL width in circular limited tokamak plasmas Nucl. Fusion 53122001

[29] Loizu J., Ricci P., Halpern F.D., Jolliet S. and Mosetto A. 2013 On the electrostatic potential in the scrape-off layer of magnetic confinement devices Plasma Phys. Control. Fusion 55124019

[30] LaBombard B. et al 2008 Critical gradients and plasma flows in the edge plasma of Alcator C-Mod Phys. Plasmas 15056106

[31] Loizu J., Ricci P., Halpern F.D., Jolliet S. and Mosetto A. 2014 Intrinsic toroidal plasma rotation in the scrape-off layer of tokamaks Phys. Plasmas 21062309

[32] Loizu J. 2013 The role of the sheath in magnetized plasma turbulence flows PhD Thesis EPFL

[33] Ricci P. and Rogers B.N. 2009 Transport scaling in interchange-driven toroidal plasmas Phys. Plasmas 16062303 